\title{
POSITIVE SOLUTIONS FOR A FOURTH ORDER DIFFERENTIAL INCLUSION WITH BOUNDARY VALUES
}

\author{
JOHN S. SPRAKER
}

Abstract. An existence result for positive solutions of a fourth order differential inclusion is proven. This is accomplished by using a certain fixed point theorem on cones and a minor extension of the Ascoli theorem. This inclusion allows both the function and its derivative on the right-hand side.

Mathematics subject classification (2010): 34B18, 34A34, 34A36, 34A60, 34B15, 47H10.

Keywords and phrases: existence of solutions, fourth order differential inclusion, fixed point, boundary value problem, Ascoli theorem.

\section{REFERENCES}

[1] R. P. Agarwal, D. O'Regan, Set valued mappings with applications in nonlinear analysis, Taylor\&Francis 2002.

[2] A. Arara, M. Benchohra, S.K. Ntouyas, A. Ouahab, Existence results for boundary value problems for fourth-order differential inclusions with nonconvex valued right hand side, Arch. Math. (Brno), 40 (2004), 219-227.

[3] J.P. Aubin, A. Cellina, Differential Inclusions, Springer 1984.

[4] J.P. Aubin, H. Frankowska, Set-valued analysis, Birkhäuser, 1990.

[5] R. Avery, J. Henderson, D.O'Regan, Functional compression-expansion fixed point theorem, Electron. J. Differ. Equ., 2008 (2008), 1-12.

[6] D.C. Biles, J.S. Spraker, Existence of positive solutions for a fourth order differential inclusion, Differ. Equ. Appl., 4 (2012), 539-546.

[7] A. Cernea, Filippov lemma for a certain differential inclusion of fourth-order, Bull. Math. Soc. Sci. Math. Roum., Nouv. Ser. 56 (104), No. 2, (2013), 181-189.

[8] L.H. Erbe, S.C. Hu, H.Y. Wang, Multiple positive solutions of some boundary value problems, J. Math. Anal. Appl., 184, (1994), 640-648.

[9] R.Y. Ma, Multiple positive solutions for a semipositone fourth-order boundary value problem, Hiroshima Math. J., 33 (2003), 217-227.

[10] R.Y. Ma, J.H. Zhang, M. Fu, The method of lower and upper solutions for fourth-order two-point boundary value problems, J. Math. Anal. Appl., 215 (1997), 415-422.

[11] D. Repovs, P.V. Semenov, Continuous Selections of Multivalued Mappings, Springer 1998.

[12] M. Svec, Periodic boundary value problems of a fourth-order differential inclusion, Arch. Math., (Brno), 33, No. 1-2, (1997), 167-171.

[13] B. Yang, Positive solutions for a fourth order boundary value problem, Electron. J. Qual. Theory Differ. Equ., 3 (2005), 1-17. 